\title{
Constrained Feedback Stabilization for Bilinear Parabolic Systems
}

\author{
Azzeddine Tsouli, Ali Boutoulout \\ TSI Team, MACS Laboratory, Faculty of Sciences, Moulay Ismail University, Meknes, Morocco \\ Email: azzeddine.tsouli@gmail.com, boutouloutali@yahoo.fr, elalamiabdessamad@gmail.com
}

Received 2 January 2015; accepted 27 March 2015; published 30 March 2015

Copyright $@ 2015$ by authors and Scientific Research Publishing Inc.

This work is licensed under the Creative Commons Attribution International License (CC BY). http://creativecommons.org/licenses/by/4.0/

(c) (i) Open Access

\begin{abstract}
In this paper, we shall study the stabilization and the robustness of a constrained feedback control for bilinear parabolic systems defined on a Hilbert state space. Then, we shall show that stabilizing such a system reduces stabilization only in its projection on a suitable subspace. For this purpose, a new constrained stabilizing feedback control that allows a polynomial decay estimate of the stabilized state is given. Also, the robustness of the considered control is discussed. An illustrating example and simulations are presented.
\end{abstract}

\section{Keywords}

Bilinear Parabolic Systems, Constrained Feedback Stabilisation, Decay Estimate, Robustness

\section{Introduction}

Bilinear systems represent a small, but important subset of nonlinear systems within which linear systems coexist as a special subclass. Adopting a bilinear model retains a well structured framework, which contains the wellknown notional concepts such as time constants and steady-state behaviour. When adopting a bilinear approach, these concepts become operation-dependent quantities which can be appropriately modelled. Bilinear system models represent an important class of nonlinear models that are defined to be linear in both state and control when considered independently, with the nonlinearity (or bilinearity) arising from coupled terms involving products of system state and control (see [1] [2]). By formulating the model appropriately, the bilinear term could also be represented by products of system output and control input, i.e. the output is defined as a system state. There are numerous combinations of product terms that could be considered, thus potentially increasing the model complexity. However, it has been found in practice that a minimal number of product terms can provide an adequate model for the purpose of control. Bilinear model structures are able to represent nonlinear phenomena more accurately than linear models, and thereby extend the range of satisfactory performance. In 
this paper, we are concerned with the question of the stabilization by a constrained feedback control for bilinear parabolic systems that can be described in the following form:

$$
\frac{\mathrm{d} y(t)}{\mathrm{d} t}=A y(t)+p(t) B y(t), \quad y(0)=y_{0}
$$

on a real Hilbert space $H$ with inner product $\langle.,$.$\rangle ; and corresponding norm \|$.$\| , where the linear operator A$ generates a contraction semigroup $(S(t))_{t \geq 0}$ on $H$ and $B \in \mathcal{L}(H)$. While the real valued function $p(.) \in L^{2}(0,+\infty ; \mathbb{R})$ represents a control. A function $y(.) \in \mathcal{C}\left(0, t_{0} ; H\right), t_{0}>0$, is a mild solution of the system (1) if and only if the solution $y(t)$ of the system (1) satisfies the variation of parameters formula:

$$
y(t)=S(t) y_{0}+\int_{0}^{t} S(t-\tau) p(\tau) B y(\tau) \mathrm{d} \tau, \quad t \geq 0
$$

(see [3]). By choosing an adequate feedback control $p(t)$ in such a way, the corresponding solution $y(t)$ of the system (1) converges to zero when $t \rightarrow+\infty$, for all $y_{0}$ in $H$. For finite-dimensional bilinear systems associated to a skew-adjoint matrix $A$, the question of stabilization has been treated in [4], under the condition:

$$
\operatorname{span}\left\{A z, a d^{0}(A, B) z, a d^{1}(A, B) z, \cdots, a d^{k}(A, B) z, \cdots\right\}=\mathbb{R}^{n}, \quad \forall z \in \mathbb{R}^{n}-\{0\}
$$

where $a d^{k}(A, B)$ is defined recursively as $a d^{0}(A, B)=B, a d^{1}(A, B)=A B-B A$ and $a d^{k+1}(A, B)=a d^{1}\left(A, a d^{k}(A, B)\right), \quad \forall k \in \mathbb{N}$.

Using the following assumptions:

$$
\langle\operatorname{Bexp}(t A) y, \exp (t A) y\rangle=0, \quad \forall t \geq 0 \Rightarrow y=0
$$

the problem of stabilization has been studied in [5]. In [3], when the linear operator $B$ is compact and $(S(t))_{t \geq 0}$ is a contraction semigroup, then using the quadratic feedback control

$$
p_{0}(t)=-\langle y(t), B y(t)\rangle
$$

a weak stabilization result is obtained under the weak observability condition:

$$
\langle B S(t) y, S(t) y\rangle=0, \quad \forall t \geq 0 \Rightarrow y=0
$$

In the case where $B$ is sequentially continuous from $H_{w}$ ( $H$ endowed with the weak topology) to $H$, the quadratic feedback control (2) weakly stabilizes the system (1), provided that the following weak observability assumption (4) holds (see [3]). Under the exact observability assumption

$$
\int_{0}^{T}|\langle B S(t) y, S(t) y\rangle| \mathrm{d} t \geq \delta\|y\|^{2}, \quad \forall y \in H,(T, \delta>0)
$$

The strong stabilization result with the following decay estimate

$$
\|y(t)\|=\mathcal{O}\left(\frac{1}{\sqrt{t}}\right), \text { as } t \rightarrow+\infty
$$

i.e. $\|y(t)\| \leq \frac{M}{\sqrt{t}}, M>0$ for $t$ large enough, has been obtained using the quadratic feedback control (5) (see [6]). However, in this way the convergence of the resulting closed loop state is not better than (8). In [7] the rational decay rates are established i.e. using the following feedback control:

$$
p_{r}(t)=-\frac{\langle y(t), B y(t)\rangle}{\|y(t)\|^{r}}, \quad r \in(-\infty, 2)
$$


It has been shown in [8] that, where the resolvent of the operator $A$ is compact, and $B$ is abounded linear, self-adjoint and monotone, the constrained feedback control law

$$
p(t)=-\frac{\langle B y(t), y(t)\rangle}{1+|\langle B y(t), y(t)\rangle|}
$$

strongly stabilizes the system (1), provided that the assumption (6) holds. It has been established in [7] that, if the linear operator $A$ generates a contraction semigroup $(S(t))_{t \geq 0}$ in $H$, then the system (1) is strongly stable with the explicit decay estimate (8), using the control (9), provided that the estimate (7) holds. Here, we will establish an explicit decay estimate of the stabilized state and the robustness of the control (9) for a large class of bilinear systems as considered in [3] [8] [9]. The method used in this paper is based on decomposing the system (1) into two suitable subsystems: the stable part and the unstable one. Then, we will show that one can concentrate on the determination of a stabilizing control for the so-called unstable part which maintains the exponential stability of the stable part. The rest of this article is as follows: in Section 2, we will give the main hypotheses that allow the decomposition of the system (1) into two subsystems. Then, under the compactness hypothesis of the operator $B$, we will give a weaker variant of the condition (6) which achieves strong stabilization of the system (1). In Section 3, we will show that under a weaker version of (6), we obtain the stabilization with the decay estimate (8). Section 4 concerns the robustness of the stabilizing controls. The last section is devoted to an illustrating example and simulations.

\section{Stabilization Results}

Let us now recall the following definition concerning the asymptotic behavior of the system (1).

\subsection{Definition}

The system (1) is weakly (resp. strongly) stabilizable if there exists a feedback control $p(t)=f(y(t)), t \geq 0$ $f: H \rightarrow K:=\mathbb{R}, \mathbb{C}$ such that the corresponding mild solution $y(t)$ of the system (1) satisfies the properties:

1. For each initial state $y_{0}$ of the system (1) there exists a unique mild solution defined for all $t \in \mathbb{R}^{+}$of the system (1),

2. $\{0\}$ is an equilibrium state of the system (1),

3. $y(t) \rightarrow 0$, weakly (resp. strongly), as $t \rightarrow+\infty$, for all $y_{0} \in H$.

In the sequel of this section, we will present an appropriate decomposition of the state space $H$ and the system (1) via the spectral properties of the operator $A$, and we apply this approach to study the stabilization problem of the system (1). In [10]-[12], it has been shown that if the spectrum $\sigma(A)$ of $A$ can be decomposed into $\sigma_{u}(A)=\{\lambda: \mathcal{R} e(\lambda) \geq-\eta, \eta>0\}$ and $\sigma_{s}(A)=\{\lambda: \mathcal{R e}(\lambda)<-\eta\}$, then the state space $H$ can be decomposed according to

$$
H=H_{u} \oplus H_{s}
$$

where $H_{u}=P_{u} H=\operatorname{vect}\left\{\varphi_{j}, 1 \leq j \leq N\right\}, H_{s}=P_{s} H=\operatorname{vect}\left\{\varphi_{j}, j>N\right\}, P_{u}$ is given by

$$
P_{u}=\frac{1}{2 \pi i} \int_{C}(\lambda I-A)^{-1} \mathrm{~d} \lambda
$$

where $C$ is a curve surrounding $\sigma_{u}(A), P_{s}=I-P_{u}$ and for all $j \geq 1, \varphi_{j}$ is the eigenvector associated to the eigenvalue $\lambda_{j}$. The projection operators $P_{u}$ and $P_{u}$ commute with $A$, and we have $A=A_{u}+A_{s}$ with $A_{u}=P_{u} A P_{u}$ and $A_{s}=P_{s} A P_{s}$. Also, for all $y(t) \in H$, we set $y_{u}=P_{u} y$ and $y_{s}=P_{s} y$. For linear systems, it has been shown that the initial system can be decomposed into two subsystems on $H_{u}$ and $H_{s}$. If $A_{s}$ satisfies the spectrum growth assumption:

$$
\lim _{t \rightarrow+\infty} \frac{\ln \left(\left\|S_{s}(t)\right\|\right)}{t}=\operatorname{supRe}\left(\sigma\left(A_{s}\right)\right)
$$


Which is equivalent to:

$$
\left\|S_{s}(t)\right\| \leq M_{1} \exp (-\eta t), \quad \forall t \geq 0 \quad \text { (for some } M_{1}>0 \text { ) }
$$

where $\left(S_{s}(t)\right)_{t \geq 0}$ denotes the semigroup generated by $A_{s}$ in $H_{s}$, then stabilizing the whole system turns out to stabilizing its projection on $H_{u}$ (see [13]). In the sequel, we suppose that the operator $B$ satisfies

$$
B H_{u} \subset H_{u} \text { and } B H_{s} \subset H_{s}
$$

It is easily verified that the condition (14) is equivalent to the fact that the linear operator $B$ commutes with $P_{u}$. We note that the condition (14) also holds in the special case: $H_{u}=H$. Let us consider that the system (1) can be decomposed in the following two subsystems:

$$
\begin{gathered}
\frac{\mathrm{d} y_{u}(t)}{\mathrm{d} t}=A_{u} y_{u}(t)+p(t) B_{u} y_{u}(t), \quad y_{u}(0)=y_{0 u} \in H_{u} \\
\frac{\mathrm{d} y_{s}(t)}{\mathrm{d} t}=A_{s} y_{s}(t)+p(t) B_{s} y_{s}(t), \quad y_{s}(0)=y_{0 s} \in H_{s}
\end{gathered}
$$

in the state spaces $H_{u}$ and $H_{s}$ respectively, and $B=B_{u} \oplus B_{s}$. It has been proved that stabilizing a linear system turns out to stabilizing its unstable part (see [13]).

\subsection{Remark}

For finite-dimensional systems, the conditions (6) and (7) are equivalent (see [5] [10]). However, in infinitedimensional case, and if $B$ is compact, then the condition (7) is impossible. Indeed, if $\left(\varphi_{j}\right)_{j>1}$ is an orthonormal basis of $H$, then applying (7) for $y=\varphi_{j}$ and using the fact that $\varphi_{j} \rightarrow 0$, weakly as $j \rightarrow+\infty$, we obtain the contradiction: $\delta=0$.

The following result concerns the strong stabilization of the system (1).

\subsection{Theorem}

Let

1. A generates a linear $C_{0}$-contraction semigroup $(S(t))_{t \geq 0}$ on $H$,

2. A allows the decomposition (10) of $H$ with $\operatorname{dim} H_{u}<+\infty$ such that (13) holds,

3. $B$ be compact such that

$$
\langle B S(t) y, S(t) y\rangle=0, \quad \forall t \geq 0 \Rightarrow y=0
$$

Then, the constrained feedback control law:

$$
p_{\rho}(t)=-\rho \frac{\langle y(t), B y(t)\rangle}{1+|\langle y(t), B y(t)\rangle|}, \quad \rho>0
$$

strongly stabilizes the system (1).

\section{Proof}

The system (1) controlled by (18) possesses a unique mild solution $y($.$) defined on a maximal interval$ $\left[0, t_{\max }[\right.$ and given by the variation of constants formula

$$
y(t)=S(t) y_{0}-\rho \int_{0}^{t} S(t-\tau) \frac{\langle y(\tau), B y(\tau)\rangle}{1+|\langle y(\tau), B y(\tau)\rangle|} B y(\tau) \mathrm{d} \tau
$$

corresponds to (18) (see [9]).

Since $(S(t))_{t \geq 0}$ is a contraction semigroup, we get:

$$
\frac{\mathrm{d}\|y(t)\|^{2}}{\mathrm{~d} t} \leq-2 \rho \frac{|\langle y(t), B y(t)\rangle|^{2}}{1+|\langle y(t), B y(t)\rangle|}, \quad \forall y_{0} \in \mathcal{D}(A)
$$


It follows from (20) that

$$
\|y(t)\| \leq\left\|y_{0}\right\|, \quad \forall t \geq 0
$$

From (19) and using the fact that $(S(t))_{t \geq 0}$ is a contraction semigroup and the Gronwall inequality, we deduce that the map $y_{0} \rightarrow y(t)$ is continuous from $H$ to $H$. Then (21) holds for all $y_{0} \in H$ by density argument, and hence $t_{\max }=+\infty$ (see [14]). Now, let us show that $y(t) \rightarrow 0$, weakly as $t \rightarrow+\infty$. Let $t_{n} \rightarrow+\infty$ such that $y\left(t_{n}\right)$ weakly converges in $H$ and let $z \in H$ such that $y\left(t_{n}\right) \rightarrow z$, weakly as $n \rightarrow \infty$. (The existence of the sequence $\left(t_{n}\right)_{n \in \mathbb{N}}$ and $z$ are ensured by (21) and by the fact that space $H$ is reflexive.) Taking $y\left(t_{n}\right)$ as initial state in (19) and using superposition property of the solution, and via the dominated convergence theorem, we obtain $\langle B S(t) z, S(t) z\rangle=0, \forall t \geq 0$. It follows from (17) that $z=0$. Hence $y(t) \rightarrow 0$, weakly as $t \rightarrow+\infty$, and since $\operatorname{dim} H_{u}<+\infty$, we have $y_{u}(t) \rightarrow 0$, as $t \rightarrow+\infty$. For the component $y_{S}(t)$ of $y(t)$ we have

$$
y_{s}(t)=S_{s}(t) y_{0 s}-\rho \int_{0}^{t} S_{s}(t-\tau) \frac{\langle y(\tau), B y(\tau)\rangle}{1+|\langle y(\tau), B y(\tau)\rangle|} B_{s} y_{s}(\tau) \mathrm{d} \tau
$$

Then for all $0 \leq t_{0} \leq t$, we have:

$$
y_{s}(t)=S_{s}\left(t-t_{0}\right) y_{s}\left(t_{0}\right)-\rho \int_{t_{0}}^{t} S_{s}(t-\tau) \frac{\langle y(\tau), B y(\tau)\rangle}{1+|\langle y(\tau), B y(\tau)\rangle|} B_{s} y_{s}(\tau) \mathrm{d} \tau
$$

It follows from (13) that

$$
\left\|y_{s}(t)\right\| \leq M_{1} \mathrm{e}^{-\eta\left(t-t_{0}\right)}\left\|y_{s}\left(t_{0}\right)\right\|+\rho M_{1}\|B\| \int_{t_{0}}^{t} \mathrm{e}^{-\eta(t-\tau)}\left\|y_{s}(\tau)\right\| \mathrm{d} \tau
$$

From Gronwall inequality, we obtain:

$$
\left\|y_{s}(t)\right\| \leq M_{1} \mathrm{e}^{\left(\rho M_{1}\|B\|-\eta\right)\left(t-t_{0}\right)}\left\|y_{s}\left(t_{0}\right)\right\|, \quad \forall t \geq t_{0}
$$

Taking $\rho<\frac{\eta}{M_{1}\|B\|}$, we deduce that $y_{s}(t) \rightarrow 0$, as $t \rightarrow+\infty$.

Hence $y(t)=y_{u}(t)+y_{s}(t) \rightarrow 0$, as $t \rightarrow+\infty$.

\section{A Decay Rate Estimate of the Stabilized State}

In what follows, we will study the strong stabilizability of the system (1) with the decay estimate (8).

Before we state our main result, the following lemmas will be needed (see [15]).

\subsection{Lemma}

Let $\left(s_{k}\right)_{k \geq 0}$ be a sequence of positive real numbers satisfying

$$
s_{k+1}+C s_{k+1}^{2+\alpha} \leq s_{k}, \quad \forall k \geq 0
$$

where $C>0$ and $\alpha>-1$ are constants. Then there exists a positive constant $M_{2}$ (depending on $\alpha$ and $C$ ) such that

$$
s_{k} \leq \frac{M_{2}}{(k+1)^{\alpha+1}}, \quad k \geq 0
$$

Let us now recall the following existing result (see [9]).

\subsection{Lemma}

Let $A$ generate a contraction semigroup $S(t)$ on $H$ and let $B$ be linear operator from $H$ into itself. 
Then the system (1), controlled by (18) possesses a unique mild solution $y(t) \in H$ for each $y_{0} \in H$ which satisfies

$$
\int_{0}^{T}|\langle S(s) y(t), B S(s) y(t)\rangle| d s=\mathcal{O}\left(\sqrt{\int_{t}^{t+T} \frac{|\langle y(s), B y(s)\rangle|^{2}}{1+|\langle B y(s), y(s)\rangle|}} d s\right), \quad \text { as } t \rightarrow+\infty
$$

For almost all $T>0$.

Our main result in this section is stated as follows:

\subsection{Theorem}

Let

1. A generates a linear $C_{0}$-semigroup $(S(t))_{t \geq 0}$ such that $\left(S_{u}(t)\right)_{t \geq 0}$ is a semigroup of isometries and (13) holds,

2. A allows the decomposition (10) of $H$ with $\operatorname{dim} H_{u}<+\infty$,

3. $B \in \mathcal{L}(H)$ such that for all $y_{u} \in H_{u}$, we have

$$
\left\langle B_{u} S_{u}(t) y_{u}, S_{u}(t) y_{u}\right\rangle=0, \quad \forall t \geq 0 \Rightarrow y_{u}=0
$$

Then the constrained feedback control law:

$$
p_{\rho, u}(t)=-\rho \frac{\left\langle y_{u}(t), B_{u} y_{u}(t)\right\rangle}{1+\left|\left\langle y_{u}(t), B_{u} y_{u}(t)\right\rangle\right|}, \quad \rho>0,
$$

strongly stabilizes the system (1) with the explicit decay estimate (8).

\section{Proof}

Let us consider the system:

$$
\frac{\mathrm{d} y_{u}(t)}{\mathrm{d} t}=A_{u} y_{u}(t)-\rho \frac{\left\langle y_{u}(t), B_{u} y_{u}(t)\right\rangle}{1+\left|\left\langle y_{u}(t), B_{u} y_{u}(t)\right\rangle\right|} B_{u} y_{u}(t), \quad y_{u}(0)=y_{0 u}
$$

Multiplying the system (29) by $y_{u}(t)$, integrating over $\Omega$ and using the fact that $\left(S_{u}(t)\right)_{t \geq 0}$ is a semigroup of isometries, we obtain:

$$
\frac{\mathrm{d}\left\|y_{u}(t)\right\|^{2}}{\mathrm{~d} t} \leq-2 \rho \frac{\left|\left\langle y_{u}(t), B_{u} y_{u}(t)\right\rangle\right|^{2}}{1+\left|\left\langle y_{u}(t), B_{u} y_{u}(t)\right\rangle\right|}
$$

which proves that the real function $t \rightarrow\left\|y_{u}(t)\right\|$ is decreasing on $\mathbb{R}^{+}$, and we have

$$
\left\|y_{u}(t)\right\| \leq\left\|y_{u}(0)\right\|, \quad \forall t \geq 0
$$

Hence, the system (29) admits a unique mild solution defined for almost all $t \geq 0$ (see [9]).

Integrating now the inequality (30) over the interval $[k T,(k+1) T]$, for $k \in \mathbb{N}$ and $T>0$, we get:

$$
\left\|y_{u}((k+1) T)\right\|^{2}-\left\|y_{u}(k T)\right\|^{2} \leq-2 \rho \int_{k T}^{(k+1) T} \frac{\left|\left\langle y_{u}(\tau), B_{u} y_{u}(\tau)\right\rangle\right|^{2}}{1+\left|\left\langle y_{u}(\tau), B_{u} y_{u}(\tau)\right\rangle\right|} \mathrm{d} \tau
$$

using now the estimate (26), we deduce that

$$
\left\|y_{u}((k+1) T)\right\|^{2}-\left\|y_{u}(k T)\right\|^{2} \leq-M\left(\int_{0}^{T}\left|\left\langle S_{u}(\tau) y_{u}, B_{u} S_{u}(\tau) y_{u}\right\rangle\right| \mathrm{d} \tau\right)^{2}
$$

for some $M>0$. Using now the fact that $\operatorname{dim}\left(H_{u}\right)<+\infty$, then the assumption (27) is equivalent to

$$
\int_{0}^{T}\left|\left\langle B_{u} S_{u}(t) y_{u}, S_{u}(t) y_{u}\right\rangle\right| \mathrm{d} t \geq \delta\left\|y_{u}\right\|^{2}, \quad \forall y_{u} \in H_{u}, \quad(T, \delta>0)
$$


From (32) and (33) we have

$$
\left\|y_{u}((k+1) T)\right\|^{2}-\left\|y_{u}(k T)\right\|^{2} \leq-M \delta^{2}\left\|y_{u}(k T)\right\|^{4}
$$

using the fact that the map $t \rightarrow\left\|y_{u}(t)\right\|$ is decreasing on $\mathbb{R}^{+}$, we obtain:

which implies that

$$
\left\|y_{u}((k+1) T)\right\|^{2}-\left\|y_{u}(k T)\right\|^{2} \leq-M \delta^{2}\left\|y_{u}((k+1) T)\right\|^{4}
$$

$$
\left\|y_{u}((k+1) T)\right\|^{2}+C\left\|y_{u}((k+1) T)\right\|^{4} \leq\left\|y_{u}(k T)\right\|^{2}, \quad C=M \delta^{2}
$$

Letting $s_{k}=\left\|y_{u}(k T)\right\|^{2}$, the last inequality can be written as

$$
s_{k+1}+C s_{k+1}^{2} \leq s_{k}, \quad \forall k \geq 0
$$

From Lemma.3.1 we have

$$
s_{k} \leq \frac{M_{2}}{k+1}
$$

For $k=\left[\frac{t}{T}\right]\left(\left[\frac{t}{T}\right]\right.$ designed the integer part of $\left.\frac{t}{T}\right)$, then we obtain $s_{k} \leq \frac{M_{3}}{t},\left(M_{3}>0\right)$, which gives

$$
\left\|y_{u}(t)\right\|^{2} \leq \frac{M_{3}}{t}
$$

Hence

$$
\left\|y_{u}(t)\right\|=\mathcal{O}\left(t^{-\frac{1}{2}}\right), \quad \text { as } t \rightarrow+\infty
$$

For the component $y_{s}(t)$, we shall show that $y_{s}(t)$ is defined for all $t \geq 0$ and exponentially converges to 0 , as $t \rightarrow+\infty$. The system (1) excited by the constrained feedback control (28) admits a unique mild solution defined for almost all $t$ in a maximal interval $\left[0, t_{\max }[\right.$ defined by

$$
y(t)=S(t) y_{0}+\int_{0}^{t} S(t-\tau) p_{\rho, u}(\tau) B y(\tau) \mathrm{d} \tau
$$

Thus

$$
y_{s}(t)=S_{s}(t) y_{0 s}+\int_{0}^{t} S_{s}(t-\tau) p_{\rho, u}(\tau) B_{s} y_{s}(\tau) \mathrm{d} \tau, \quad \forall t \in\left[0, t_{\max }[\right.
$$

It follows from (13) that

$$
\left\|y_{s}(t)\right\| \leq M_{1} \mathrm{e}^{-\eta t}\left\|y_{0 s}\right\|+\rho M_{1}\|B\| \int_{0}^{t} \mathrm{e}^{-\eta(t-\tau)}\left\|y_{s}(\tau)\right\| \mathrm{d} \tau
$$

For almost all $t \in\left[0, t_{\max }[\right.$. The Gronwall inequality then yields:

$$
\left\|y_{s}(t)\right\| \leq M_{1}\left\|y_{0 s}\right\| \mathrm{e}^{\left(\rho M_{1}\|B\|-\eta\right) t}, \quad \forall t \geq 0
$$

Taking $\rho<\frac{\eta}{M_{1}\|B\|}$, it follows from (36) that $y_{s}(t)$ is bounded on $\left[0, t_{\max }\left[\right.\right.$ so $t_{\max }=+\infty$, and therefore (36) holds for all $t \geq 0$. Hence, from (34) and (36), the solution of (1) satisfies the estimate (8). This completes the proof of Theorem 3.3.

\subsection{Remark}

1. Since the function $t \rightarrow\left\|y_{u}(t)\right\|$ decreasing in $\mathbb{R}^{+}$, we have 


$$
\exists t_{0} \geq 0 ; \quad y_{u}\left(t_{0}\right)=0 \Leftrightarrow y_{u}(t)=0, \quad \forall t \geq t_{0}
$$

In this case, we have

$$
p_{\rho, u}(t)=0, \quad \forall t \geq t_{0} \Rightarrow y(t)=S_{s}\left(t-t_{0}\right) y_{s}\left(t_{0}\right), \quad \forall t \geq t_{0}
$$

Hence, using (13) the system (1) is exponentially stable.

2. The constrained feedback control (28) depends only on the unstable part $y_{u}(t)$ and we have

$$
\left|p_{\rho, u}(t)\right|<\rho, \quad \forall t \geq 0
$$

3. The constrained feedback control (28) satisfies

$$
\left|p_{\rho, u}(t)\right|=\mathcal{O}\left(\frac{1}{t}\right), \quad \text { as } t \rightarrow+\infty
$$

4. We note that (27) is weaker than (6). The converse is not true as we can see taking an orthonormal basis $\left(\phi_{n}\right)_{n \geq 1}$ of $H, A z=-\left\langle z, \phi_{1}\right\rangle \phi_{1}$ and $B z=\sum_{n=1}^{+\infty} \frac{\left\langle z, \phi_{n}\right\rangle}{n^{2}} \phi_{n}$.

5. In the case $\operatorname{dim} H_{u}=+\infty$ and $B$ is nonlinear and locally Lipschitz, such that $B(0)=0$, then using the same techniques as in [9], we can obtain the result of Theorem 3.3, if the estimate (7) is changed to (33).

\section{Robustness}

In this section, we study the robustness of the controls (18) and (28), under a class of perturbations of the system (1).

\subsection{Strong Robustness}

In this part, we consider the strong robustness of the feedback (18). Then, we will show that the stability property of the system (1) remains invariant under a certain class of bounded perturbations.

Let us consider the following perturbed system

$$
\frac{\mathrm{d} y(t)}{\mathrm{d} t}=A y(t)+p(t) B y(t)+\xi(y(t)), \quad y(0)=y_{0}
$$

where the linear bounded operator $\xi=\xi_{u}+\xi_{s}$ is such that the system (37) is decomposed into two following subsystems:

$$
\begin{array}{cl}
\frac{\mathrm{d} y_{u}(t)}{\mathrm{d} t}=A_{u} y_{u}(t)+p(t) B_{u} y_{u}(t)+\xi_{u}\left(y_{u}(t)\right), & y_{u}(0)=y_{0 u} \in H_{u} \\
\frac{\mathrm{d} y_{s}(t)}{\mathrm{d} t}=A_{s} y_{s}(t)+p(t) B_{s} y_{s}(t)+\xi_{s}\left(y_{u}(t)\right), & y_{s}(0)=y_{0 s} \in H_{s}
\end{array}
$$

The following main result concerns the strong stability of the system (37).

\subsection{Proposition}

Let

1. A generates a linear $C_{0}$-contraction semigroup $(S(t))_{t \geq 0}$ on $H$ such that (13) holds,

2. The operator $B$ is compact such that (6) holds,

3. The linear operator $\xi$ is compact and satisfying $\left\|\xi_{s}\right\|<\frac{\eta}{M_{1}}$ and $\langle\xi(y), y\rangle \leq 0, \forall y \in H$.

Then the system (37) is strongly stabilizable.

\section{Proof}

First, let us note that 0 remains an equilibrium state of the perturbed system (37), which can be written in the form: 


$$
\frac{\mathrm{d} y(t)}{\mathrm{d} t}=A y(t)+g(y(t)), \quad y(0)=y_{0}
$$

where $g=f+\xi$ and $f(y)= \begin{cases}-\frac{\rho\langle B y, y\rangle}{1+|\langle B y, y\rangle|} B y, & y \neq 0 ; \\ 0, & y=0 .\end{cases}$

Since $f$ and $\xi$ are locally Lipschitz, so is $g$. Also $g$ is dissipative: $\langle\xi(y), y\rangle \leq 0, \forall y \in H$.

The assumption $\langle\xi(y), y\rangle \leq 0, \forall y \in H$, together with (6) guarantees the following implication

$$
\langle g(S(t) y), S(t) y\rangle=0 \Rightarrow y=0
$$

Then the weak stability of the perturbed system (37) follows from Theorem 2.4 of Ball [3], and since $\operatorname{dim} H_{u}<+\infty$, we have $y_{u}(t) \rightarrow 0$, as $t \rightarrow+\infty$. For the component $y_{s}(t)$ of the solution $y(t)$ of the system (37), and for all $0 \leq t_{0} \leq t$, we have:

$$
y_{s}(t)=S_{s}\left(t-t_{0}\right) y_{s}\left(t_{0}\right)-\rho \int_{t_{0}}^{t} S_{s}(t-\tau) \frac{\langle y(\tau), B y(\tau)\rangle}{1+|\langle y(\tau), B y(\tau)\rangle|} B_{s} y_{s}(\tau) \mathrm{d} \tau+\int_{t_{0}}^{t} S_{s}(t-\tau) \xi_{s}\left(y_{s}(\tau)\right) \mathrm{d} \tau
$$

It follows from (13) that

$$
\left\|y_{s}(t)\right\| \leq M_{1} \mathrm{e}^{-\eta\left(t-t_{0}\right)}\left\|y_{s}\left(t_{0}\right)\right\|+\left(\rho M_{1}\|B\|+M_{1}\left\|\xi_{s}\right\|\right) \int_{t_{0}}^{t} \mathrm{e}^{-\eta(t-\tau)}\left\|y_{s}(\tau)\right\| \mathrm{d} \tau
$$

From Gronwall inequality, we obtain:

$$
\left\|y_{s}(t)\right\| \leq M_{1} \mathrm{e}^{\left(\rho M_{1}\|B\|+M_{1}\left\|\xi_{s}\right\|-\eta\right)\left(t-t_{0}\right)}\left\|y_{s}\left(t_{0}\right)\right\|, \quad \forall t \geq t_{0}
$$

Taking $\rho<\frac{\eta-M_{1}\left\|\xi_{s}\right\|}{M_{1}\|B\|}$, we obtain $y_{s}(t) \rightarrow 0$, as $t \rightarrow+\infty$. Hence, the solution $y(t)$ of the system (37) strongly converges to 0 , as $t \rightarrow+\infty$.

\subsection{A Polynomial Decay Estimate for the Perturbed System}

Our second main result in this section is stated as follows:

\subsection{Proposition}

Let

1. A generate a linear $C_{0}$-semigroup $(S(t))_{t \geq 0}$ such that $\left(S_{u}(t)\right)_{t \geq 0}$ is a semigroup of isometries and (13) holds,

2. A allows the decomposition (10) of $H$ with $\operatorname{dim} H_{u}<+\infty$,

3. $B \in \mathcal{L}(H)$ satisfies (27),

4. $\left\|\xi_{s}\right\| \leq \frac{\eta}{M_{1}}$ and $\left\langle\xi_{u}(y), y\right\rangle \leq 0$, for all $y \in H_{u}$.

Then the constrained feedback control (28) strongly stabilizes the system (37) with the explicit decay estimate (8).

Proof

Let us consider the system:

$$
\frac{\mathrm{d} y_{u}(t)}{\mathrm{d} t}=A_{u} y_{u}(t)-\rho \frac{\left\langle y_{u}(t), B_{u} y_{u}(t)\right\rangle}{1+\left|\left\langle y_{u}(t), B_{u} y_{u}(t)\right\rangle\right|} B_{u} y_{u}(t)+\xi_{u}\left(y_{u}(t)\right), \quad y_{u}(0)=y_{0 u}
$$

Multiplying the system (43) by $y_{u}(t)$ and integrating over $\Omega$ and using the fact that $\left(S_{u}(t)\right)_{t \geq 0}$ is a se- 
migroup of isometries and the hypothesis: $\left\langle\xi_{u}\left(y_{u}\right), y_{u}\right\rangle \leq 0, \forall y_{u} \in H_{u}$, we obtain:

$$
\frac{\mathrm{d} \|\left. y_{u}(t)\right|^{2}}{\mathrm{~d} t} \leq-2 \rho \frac{\left|\left\langle y_{u}(t), B_{u} y_{u}(t)\right\rangle\right|^{2}}{1+\left|\left\langle y_{u}(t), B_{u} y_{u}(t)\right\rangle\right|}
$$

which gives $\left\|y_{u}(t)\right\| \leq\left\|y_{u}(0)\right\|, \quad \forall t \geq 0$. Then, the system (43) admits a unique global mild solution $y(t)$ defined for almost all $t \geq 0$. By the same argument as in the proof of Theorem 3.3, the solution $y(t)$ of the system (37) satisfies:

$$
\|y(t)\|=\mathcal{O}\left(t^{-\frac{1}{2}}\right), \quad \text { as } t \rightarrow+\infty
$$

which completes the proof of Proposition 4.2.

\section{Application and Simulations}

\subsection{An Application}

In this part, we will give an illustrating example of the established results.

Example

Let us consider the following 1-d bilinear heat equation:

$$
\begin{cases}\frac{\partial y(x, t)}{\partial t}=\frac{\partial^{2} y(x, t)}{\partial x^{2}}+p(t) B y(t), & x \in(0,1), t>0, \\ \frac{\partial y(0, t)}{\partial x}=\frac{\partial y(1, t)}{\partial x}=0, & \forall t>0,\end{cases}
$$

where $y(t)$ is the temperature profile at time $t$. We suppose that the system is controlled via the flow of a liquid $p(t)$ in an adequate metallic pipeline. Here we take the state space $H=L^{2}(0,1)$ and the operator $A$ is defined by $A y=\frac{\partial^{2} y}{\partial x^{2}}$, with $\mathcal{D}(A)=\left\{y \in H^{2}(0,1) \mid \frac{\partial y(0, t)}{\partial x}=\frac{\partial y(1, t)}{\partial x}=0\right\}$. The domain of $A$ gives the homogeneous Neumann boundary condition imposed at the ends of the bar which require specifying how the heat flows out of the bar and means that both ends are insulated. The spectrum of $A$ is given by the simple eigenvalues $\lambda_{j}=-\pi^{2}(j-1)^{2}, j \in \mathbb{N}^{*}$ and eigenfunctions $\varphi_{1}(x)=1$ and $\varphi_{j}(x)=\sqrt{2} \cos ((j-1) \pi x)$ for all $j \geq 2$. Then the subspace $H_{u}$ is the one-dimensional space spanned by the eigenfunction $\varphi_{1}$, and we have $S_{u}(t) y_{u}=\left\langle y_{u}, \varphi_{1}\right\rangle \varphi_{1}$ so $S_{u}(t)=I_{H_{u}}$ (the identity) and hence $\left(S_{u}(t)\right)_{t \geq 0}$, is a semigroup of isometries. The operator of control $B$, is defined by: $B y=\sum_{j=1}^{+\infty} \alpha_{j}\left\langle y, \varphi_{j}\right\rangle \varphi_{j}, \quad \alpha_{j} \geq 0, \forall j \geq 1$, such that $\sum_{j=1}^{+\infty} \alpha_{j}^{2}<\infty$ (see [16]). From the relation: $\left\langle B_{u} S_{u}(t) y_{u}, S_{u}(t) y_{u}\right\rangle=\alpha_{1}\left|\left\langle y_{u}, \varphi_{1}\right\rangle\right|^{2}$, we can see that (27) holds if $\alpha_{1}>0$. To examine the estimate (8), remarking for the scalar functions $y_{j}(t)=\left\langle y(t), \varphi_{j}\right\rangle, \forall j \geq 1$ we have

$$
\frac{\mathrm{d} y_{1}(t)}{\mathrm{d} t}=\frac{\mathrm{d} y_{u}(t)}{\mathrm{d} t}=-\frac{\rho \alpha_{1} y_{u}^{3}(t)}{1+\alpha_{1} y_{u}^{2}(t)}, \quad \forall t \geq 0
$$

which implies that

$$
\frac{\mathrm{d}}{\mathrm{d} t} y_{u}^{2}(t)=-\frac{2 \rho \alpha_{1} y_{u}^{4}(t)}{1+\alpha_{1} y_{u}^{2}(t)}
$$


Letting $x(t)=y_{u}^{2}(t)$, we obtain

$$
x^{\prime}(t) x^{-2}(t)=-\frac{2 \rho \alpha_{1}}{1+\alpha_{1} x(t)}, \quad \forall t>0
$$

Integrating now the last equality from 0 to $t$, we get

$$
x^{-1}(t)-x^{-1}(0)=\int_{0}^{t} \frac{2 \rho \alpha_{1}}{1+\alpha_{1} x(\tau)} \mathrm{d} \tau
$$

from (47), we deduce that the nonnegative scalar map $t \rightarrow x(t)$ is decreasing for all $t \geq 0$, and we have

$$
x^{-1}(t) \geq \frac{2 \rho \alpha_{1} t}{1+\alpha_{1} x(0)}, \quad \forall t>0
$$

which means that

$$
\left|y_{u}(t)\right| \leq \sqrt{\frac{1+\alpha_{1} y_{0 u}^{2}}{2 \rho \alpha_{1} t}}, \quad \forall t>0, \quad y_{0 u} \neq 0
$$

Then

$$
\left|y_{u}(t)\right|=\mathcal{O}\left(\frac{1}{\sqrt{t}}\right), \quad \text { as } t \rightarrow+\infty
$$

Furthermore, the control in this case is defined by

$$
p_{\rho, u}(t)=-\frac{\rho \alpha_{1} y_{u}^{2}(t)}{1+\alpha_{1} y_{u}^{2}(t)}, \quad \forall t \geq 0
$$

For $j \geq 2$, the functions $y_{j}(t)$ are characterized by $y_{j}(0)=\left\langle y(0), \varphi_{j}\right\rangle, \forall j \geq 2$ and satisfy

$$
\frac{\partial}{\partial t} y_{j}(t)=\left(\lambda_{j}-\frac{\rho \alpha_{1} \alpha_{j} y_{1}^{2}(t)}{1+\alpha_{1} y_{1}^{2}(t)}\right) y_{j}(t), \quad t \geq 0
$$

which implies that

$$
\left|y_{j}(t)\right| \leq \mathrm{e}^{-\pi^{2}(j-1)^{2} t}\left|y_{j}(0)\right|, \quad j \geq 2
$$

Then

$$
\left\|y_{s}(t)\right\| \leq \mathrm{e}^{-\pi^{2} t}\left\|y_{s}(0)\right\|, \quad \forall t \geq 0
$$

Hence, the system (45) is strongly stable with the decay rate estimate (8).

Let us reconsider the above example with the perturbation $\xi$ defined by:

$$
\xi(y)=-\beta y, \quad \beta \in\left(0, \frac{\pi^{2}}{2}\right), \quad \forall y \in H
$$

It is clear that the function $\xi$ satisfies the conditions of Proposition 4.2.1. Then the perturbed closed-loop system remains stable, i.e., the control (48) still stabilizes the perturbed system i.e., the control (48) is strongly robust.

\subsection{Simulations}

In this part, taking in the system (45), the operator $B=I$ and $y_{0}(x)=x+0.3$.

Then we obtain the results shown in Figures 1-5. 


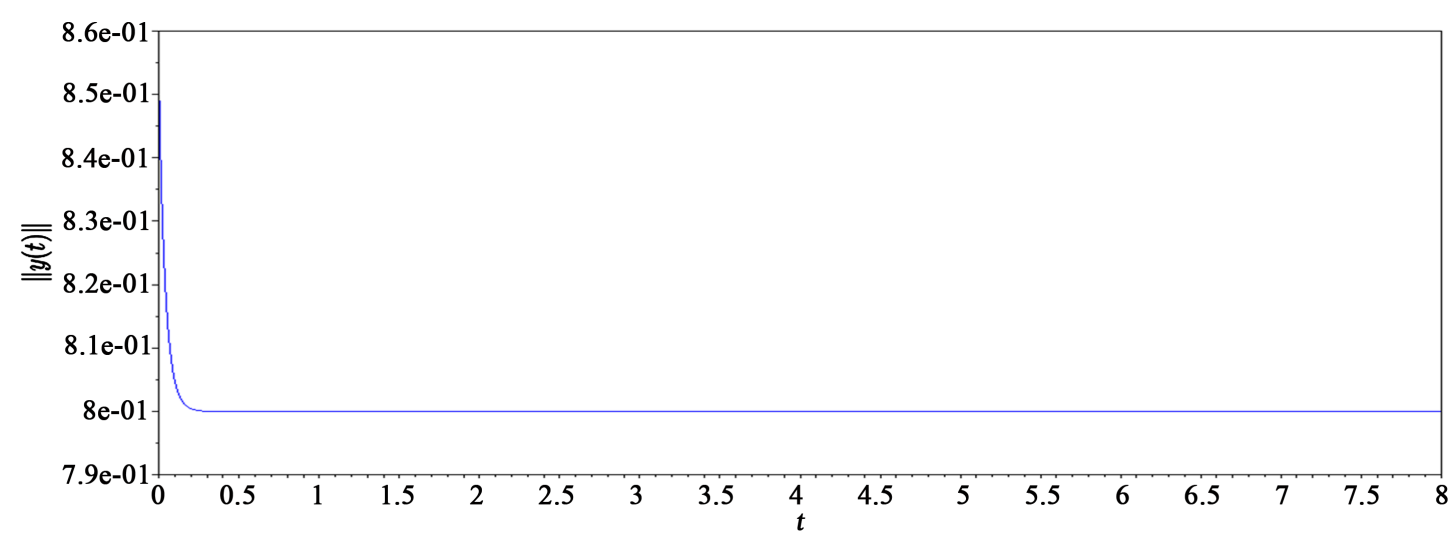

Figure 1. The norm of the free state.

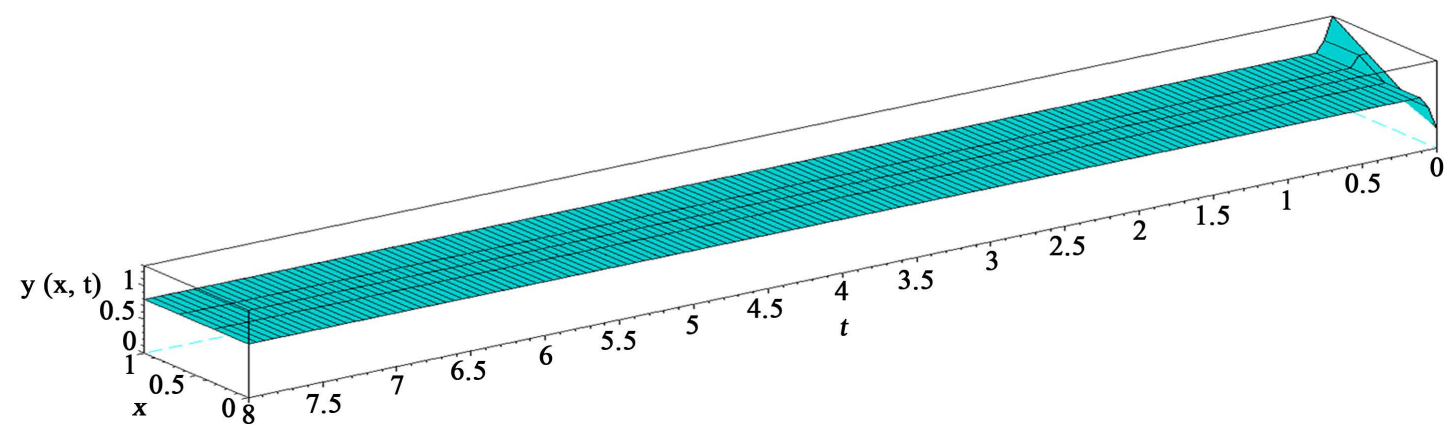

Figure 2. The free state.

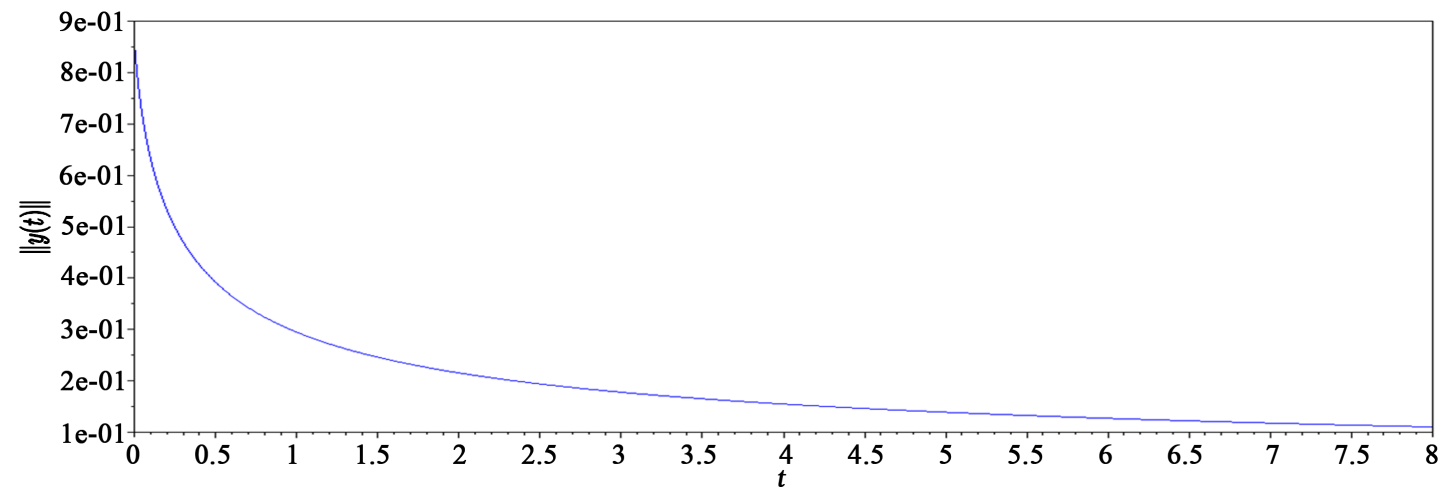

Figure 3. The norm of the stabilized state.

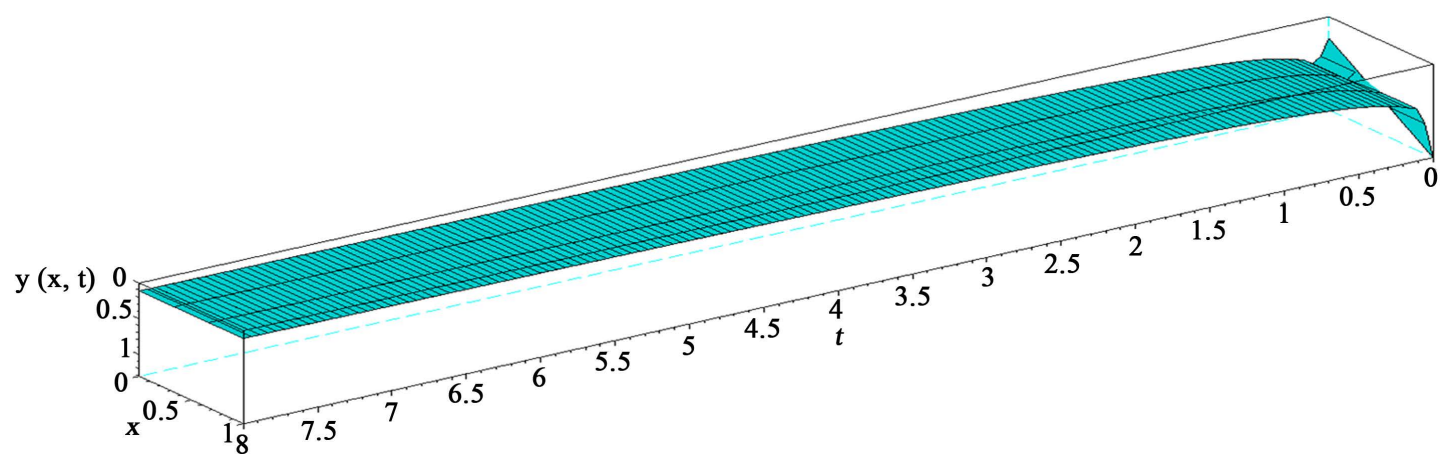

Figure 4. The stabilized state. 


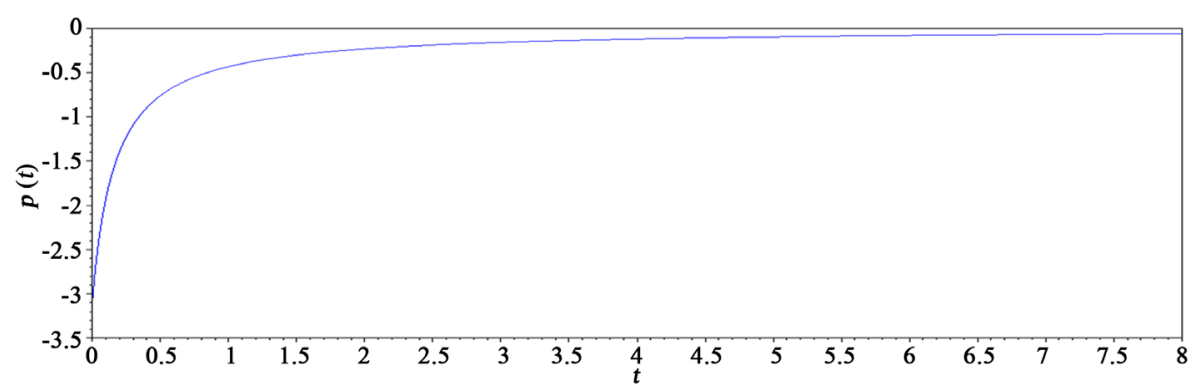

Figure 5. The stabilizing control.

\section{Conclusion}

In this work, we have considered the problem of strong stabilization with polynomial decay rate of the stabilized state for bilinear parabolic systems that can be decomposed in the stable and unstable parts (15) and (16) under a weaker condition (27). We have also considered the problem of using a stabilizing feedback control for the unstable part (15) only that can make the whole system (1) stable. Various questions remain open. This is the case of stabilization for nonlinear systems. Finally, we have studied the robustness problem of the stabilizing controls with respect to a class of perturbations, but a confrontation to more realistic situations remain done. This leads us to consider the stabilization problem for stochastic bilinear system.

\section{References}

[1] Mohler, R.R. (1973) Bilinear Control Process. Academic Press, New York.

[2] Tsouli, A. and Boutoulout, A. (2014) Controllability of the Parabolic System via Bilinear Control. Journal of Dynamical and Control Systems, 20, in press. http://dx.doi.org/10.1007/s10883-014-9247-2

[3] Ball, J. and Slemrod, M. (1979) Feedback Stabilization of Distributed Semilinear Control Systems. Applied Mathematics and Optimization, 5, 169-179. http://dx.doi.org/10.1007/BF01442552

[4] Jurdjevic, V. and Quinn, J.P. (1978) Controllability and Stability. Journal of Deferential Equations, 28, 381-389. http://dx.doi.org/10.1016/0022-0396(78)90135-3

[5] Quinn, J.P. (1980) Stabilization of Bilinear Systems by Quadratic Feedback Control. Journal of Mathematical Analysis and Applications, 75, 66-80. http://dx.doi.org/10.1016/0022-247X(80)90306-6

[6] Ouzahra, M. (2008) Strong Stabilization with Decay Estimate of Semilinear Systems. Systems and Control Letters, 57, 813-815. http://dx.doi.org/10.1016/j.sysconle.2008.03.009

[7] Ouzahra, M., Tsouli, A. and Boutoulout, A. (2012) Stabilization and Polynomial Decay Estimate for Distributed Semilinear Systems. International Journal of Control, 85, 451-456. http://dx.doi.org/10.1080/00207179.2012.656144

[8] Bounit, H. and Hammouri, H. (1999) Feedback Stabilization for a Class of Distributed Semilinear Control Systems. Nonlinear Analysis, 37, 953-969. http://dx.doi.org/10.1016/S0362-546X(97)00577-4

[9] Tsouli, A., Ouzahra, M. and Boutoulout, A. (2014) A Decay Estimate for Constrained Semilinear Systems. Information Sciences Letters, 3, 77-83. http://dx.doi.org/10.12785/isl/030206

[10] Chen, M.S. (1998) Exponential Stabilization of a Constrained Bilinear System. Automatica, 34, 989-992. http://dx.doi.org/10.1016/S0005-1098(98)00037-5

[11] Kato, T. (1980) Perturbation Theory for Linear Operators. Springer, New York.

[12] Ouzahra, M. (2011) Feedback Stabilization of Parabolic Systems with Bilinear Controls. Electronic Journal of Differential Equations, 38, 1-10.

[13] Triggiani, R. (1975) On the Stabilizability Problem in Banach Space. Journal of Mathematical Analysis and Applications, 52, 383-403. http://dx.doi.org/10.1016/0022-247X(75)90067-0

[14] Pazy, A. (1983) Semi-Groups of Linear Operators and Applications to Partial Deferential Equations. Springer Verlag, New York. http://dx.doi.org/10.1007/978-1-4612-5561-1

[15] Ammari, K. and Tucsnak, M. (2001) Stabilization of Second Order Evolution Equations by a Class of Unbounded Feedbacks. ESAIM: Control, Optimisation and Calculus of Variations, 6, 361-386. http://dx.doi.org/10.1051/cocv:2001114

[16] Ouzahra, M. (2010) Exponential and Weak Stabilization of Constrained Bilinear Systems. SIAM Journal on Control and Optimization, 48, 3962-3974. http://dx.doi.org/10.1137/080739161 